\title{
Length thresholds for graphic lists given fixed largest and smallest entries and bounded gaps
}

\author{
Michael D. Barrus, Stephen G. Hartke, Kyle F. Jao, Douglas B. West
}

\begin{abstract}
In a list $\left(d_{1}, \ldots, d_{n}\right)$ of positive integers, let $r$ and $s$ denote the largest and smallest entries. A list is gap-free if each integer between $r$ and $s$ is present. We prove that a gapfree even-summed list is graphic if it has at least $r+\frac{r+s+1}{2 s}$ terms. With no restriction on gaps, length at least $\frac{(r+s+1)^{2}}{4 s}$ suffices, as proved by Zverovich and Zverovich. Both bounds are sharp within 1 . When the gaps between consecutive terms are bounded by $g$, we prove a more general length threshold that includes both of these results. As a tool, we prove that if an even-summed positive list $d$ has no repeated entries other than $r$ and $s$ (and the length exceeds $r$ ), then to prove that $d$ is graphic it suffices to check only the $\ell$ th Erdős-Gallai inequality, where $\ell=\max \left\{k: d_{k} \geq k\right\}$.
\end{abstract}

\section{Introduction}

A list of integers is graphic if it is the list of vertex degrees for some graph (with no loops or multiple edges). We consider only nonincreasing positive lists, writing a list $d$ as $\left(d_{1}, \ldots, d_{n}\right)$ with $d_{1} \geq \cdots \geq d_{n}$. Such a list is gap-free if it has entries with all values between the largest entry $r$ and the smallest entry $s$; it is even-summed if $\sum_{i=1}^{n} d_{i}$ is even. We define the gap at $i$ in a list $d$ to be $d_{i}-d_{i+1}$. A list with $r=s$ is graphic if it has even sum and $n>r$ (realized using edge-disjoint spanning cycles or 1-factors, depending on whether $n$ is odd or even). The same conclusion also holds when $r-s=1$ (see [9]). Among even-summed lists with largest entry $r$, smallest entry $s$, and all gaps at most $g$, seek the least $n$ such that every such list with length at least $n$ is graphic; we determine it within 1 .

Graphic lists have many characterizations. Erdős and Gallai [3] proved in 1960 that a list $d$ is graphic if and only if it has even sum and satisfies $L_{k}(d) \leq R_{k}(d)$ for each integer $k$ with $1 \leq k \leq n$, where $L_{k}(d)=\sum_{i=1}^{k} d_{i}$ and $R_{k}(d)=k(k-1)+\sum_{i=k+1}^{n} \min \left\{k, d_{i}\right\}$. In fact, Zverovich and Zverovich [10] proved that it suffices to check the first $\ell$ of these inequalities, where $\ell=\max \left\{k: d_{k} \geq k\right\}$ (see [5, 4] for a slightly weaker statement). Eggleton [2] (and later Tripathi and Vijay [8]) proved the stronger statement that it suffices to check only 
the inequalities for the last index having each value in the list. Values between $r$ and $s$ are internal values. As a tool in our argument, we prove in Section 3 that if a list has no repeated internal values (and $n>r$ ), then it suffices to check only the $\ell$ th inequality. The conclusion also holds when there is exactly one instance of two consecutive equal internal values, and this is sharp.

We approach our problem by finding a length threshold for lists to satisfy the ErdösGallai inequalities, which we henceforth call the E-G inequalities. We use the Aigner-Triesch method. In Section 2, we describe the use of this method to prove sufficiency of conditions for lists to be graphic. The method introduces an order relation $P$ on the set of lists with fixed sum and reduces the problem to proving that lists that are maximal in $P$ among those satisfying the condition are graphic. We further reduce the problem by comparing these maximal lists from the sets with various sums, reducing sufficiency to the study of certain key lists.

Let $D_{n}(r, s, g)$ denote the set of nonincreasing nonnegative integer lists with length $n$, largest entry $r$, smallest entry $s$, and all gaps at most $g$. The case $g=0$ requires only $n>r$, as mentioned in the first paragraph, so we henceforth restrict to $g>0$ and consider only $r>s$. The first and last nonzero gaps in a list are external gaps (they may be at the same position); gaps between them are internal gaps. A list in $D_{n}(r, s, g)$ is $g$-uniform if all internal and external gaps except possibly the last one equal $g$. We show in Section 2 that to prove sufficiency of the length threshold for lists with gaps at most $g$, it suffices to prove it sufficient for $g$-uniform lists. The resulting sharpness examples are $g$-uniform.

Call a list feasible if it satisfies the E-G inequalities. In terms of $r, s$, and $g$, we obtain a sharp threshold $h(r, s, g)$ such that when $n \geq h(r, s, g)$, every list in $D_{n}(r, s, g)$ is feasible; the argument that proves the threshold sufficient also constructs an infeasible list when $n$ is smaller. The general expression for $h(r, s, g)$ is obtained in Theorem 4.2. When $g$ divides $r-s$, the formula for $h(r, s, g)$ simplifies to

$$
\frac{1}{s}\left(\left\lfloor\frac{(r+s)^{2}}{4}\right\rfloor+\left\lceil\frac{r+s}{2}\right\rceil-\frac{g}{2}\left\lfloor\frac{(r-s)^{2}}{2 g^{2}}\right\rfloor\right) .
$$

Depending on the parameters $r, s, g$, the infeasible list given for $n=\lceil h(r, s, g)\rceil-1$ may have odd sum. In this case, the length threshold sufficient for even-summed lists with specified maximum, minimum, and bound on gaps to be graphic may be smaller by 1 than the threshold we give for feasibility. We show that the difference between the threshold lengths for feasible lists and graphic lists is never more than 1 , and we present a family with $g=1$ where the thresholds do differ by 1. 
The extreme cases for $g$ hold particular interest. When $g=1$, the threshold for feasibility reduces to $r+\frac{r+s+\epsilon}{2 s}$ (linear in $r$ ), where $\epsilon=0$ if $r+s$ is even and $\epsilon=1$ if $r+s$ is odd. This is the most severe restriction on gaps. The other end of the spectrum is $g=r-s$, which means that no gap restriction is imposed. Here the threshold reduces to $\frac{(r+s+1)^{2}-\epsilon^{\prime}}{4 s}$ (quadratic in $r$ ), where $\epsilon^{\prime}=0$ if $r+s$ is odd and $\epsilon^{\prime}=1$ if $r+s$ is even. Zverovich and Zverovich [10] showed that $\frac{(r+s+1)^{2}}{4 s}$ suffices.

\section{Maximal List for Fixed $n, r, s, g$, and Sum}

By the Erdős-Gallai Theorem, a list is graphic if and only if it is feasible and has even sum. In light of this theorem, using the Aigner-Triesch method [1] to show sufficiency of conditions for feasibility will also give sufficient conditions for lists to be graphic. This allows us to ignore the parity of the degree sum in applying this method. Let $R$ be a family of lists (for example, the graphic lists or the feasible lists). The Aigner-Triesch method for proving that a condition $Q$ is sufficient for membership in $R$ consists of three steps:

1. Define a poset $P$ on the set of lists (usually with fixed sum) and show that the elements of $P$ belonging to $R$ form an ideal (a downward-closed set) in $P$.

2. Determine the maximal elements of $P$ among those satisfying $Q$.

3. Prove that these maximal elements are in $R$.

For lists with fixed sum, an order relation often used in applying the Aigner-Triesch method is the dominance order, which puts $d \leq d^{\prime}$ if $\sum_{i=1}^{k} d_{i} \leq \sum_{i=1}^{k} d_{i}^{\prime}$ for all $k$ (trailing terms are assumed to be 0). For the dominance order on a set of lists with fixed sum, the proof of Step 1 when $R$ is the family of graphic lists is immediate and is used in [1]; we present the corresponding argument in Lemma 2.1 for the family of feasible lists.

When Step 1 holds for a given poset, it also holds for any subposet. Let $P_{m}$ be the dominance order on nonincreasing nonnegative integer lists with sum $m$. After proving Step 1 for $P_{m}$, we will consider subposets of the form $P_{m, n, r, s, g}$, fixing the sum $m$, length $n$, largest entry $r$, positive smallest entry $s$, and bound $g$ on all gaps. Since $g$ is only a bound on the largest gap, these subposets are not disjoint. Nevertheless, Step 1 will hold for each such subposet. The condition $Q$ we want to prove sufficient is a lower bound on the length $n$; therefore, in $P_{m, n, r, s, g}$ all lists or no lists satisfy $Q$. For Step 2, we prove that $P_{m, n, r, s, g}$ has a unique maximal element. We then show that the maximal element of $P_{m, n, r, s, g}$ is feasible when the length threshold in terms of the parameters $r, s$, and $g$ is satisfied. To do this, 
we compare the maximal elements for distinct values of $m$, thus reducing the problem to showing feasibility for the maximal element of certain key subposets.

We begin with Step 1 for $P_{m}$. All lists are nonincreasing. Shifting a unit from $i$ to $j$ in a list $d$ produces another nonincreasing list $d^{\prime}$ that agrees with $d$ in all positions except $i$ and $j$, and in those positions $d_{i}^{\prime}=d_{i}-1$ and $d_{j}^{\prime}=d_{j}+1$. The unit is shifted later if $i<j$, otherwise earlier. In a poset, an element $x$ covers an element $y$ if $y<x$ and there is no element $z$ such that $y<z<x$. To prove Step 1, we show (1) if $d$ covers $d^{\prime}$ in $P_{m}$, then one unit can be shifted later in $d$ to obtain $d^{\prime}$, and (2) shifting one unit later preserves feasibility.

Lemma 2.1. If $d$ covers $d^{\prime}$ in $P_{m}$ and $d$ is feasible, then $d^{\prime}$ is feasible.

Proof. We obtain $d^{\prime}$ from $d$ by shifting a unit later. Let $i$ and $j$ be the first and last positions where $d$ and $d^{\prime}$ differ. Since $d>d^{\prime}$, we have $d_{i}>d_{i}^{\prime}$ and $d_{j}<d_{j}^{\prime}$. Choose $p$ and $q$ to minimize $q-p$ such that $i \leq p<q \leq j$ and $d_{p}>d_{p}^{\prime}$ and $d_{q}<d_{q}^{\prime}$. Form $\hat{d}$ by shifting a unit from position $p$ to position $q$ in $d$. Since $d>\hat{d} \geq d^{\prime}$ in $P_{m}$, we obtain $\hat{d}=d^{\prime}$.

Hence it suffices to prove that shifting a unit later preserves feasibility. Suppose that $d^{\prime}$ is obtained from $d$ by shifting a unit from $i$ to $j$ with $i<j$. We compare the $k$ th E-G inequalities for $d$ and $d^{\prime}$. Since we shifted a unit later, $L_{k}\left(d^{\prime}\right) \leq L_{k}(d)$.

The only position that can contribute less to $R_{k}\left(d^{\prime}\right)$ than to $R_{k}(d)$ (smaller by 1 ) is position $i$, and it does so only when $d_{i} \leq k<i$. Since we shifted later from $i$ to $j$, also $d_{j}<d_{i} \leq k<j$, and hence in this case position $j$ contributes more to $R_{k}\left(d^{\prime}\right)$ than to $R_{k}(d)$. Thus $R_{k}\left(d^{\prime}\right) \geq R_{k}(d)$ for all $k$, and feasibility of $d$ implies feasibility of $d^{\prime}$.

Lemma 2.2. The poset $P_{m, n, r, s, g}$ has a unique maximal element. In it, there is at most one internal gap that is less than $g$, and if there is such a gap it is $g-1$.

Proof. Let $d$ be a maximal element. If $d$ has at least two internal gaps that are less than $g$, then let $i$ and $j$ be the positions of the first and last such gaps. Form $d^{\prime}$ by shifting a unit from $j+1$ to $i$ (earlier). The gaps at $i$ and $j$ increase by 1 , but they are still at most $g$. The gaps at $i-1$ and $j+1$ decrease by 1 , but the choice of $i$ and $j$ implies that they were external or were equal to $g$ before the shift. In either case, the list $d^{\prime}$ is nonincreasing and belongs to $P_{m, n, r, s, g}$. Since $d^{\prime}>d$, this is a contradiction.

Hence at most one internal gap is less than $g$, say at $j$. If it is less than $g-1$, then define $d^{\prime}$ by shifting a unit from $j+1$ to $j$. The gap at $j$ grows by 2 , and the gaps at $j-1$ and $j+1$ (which were external or equal to $g$ ) are smaller by 1 . Again $d^{\prime} \in P_{m, n, r, s, g}$ and $d^{\prime}>d$.

It remains to prove that only one element of $P_{m, n, r, s, g}$ can have the properties obtained above for all maximal elements. Suppose that $d$ and $d^{\prime}$ are distinct maximal elements of 
$P_{m, n, r, s, g}$. Let $i$ be the first index where $d$ and $d^{\prime}$ differ, named so that $d_{i}^{\prime}<d_{i}$. Let $k$ be the last index such that $d_{k}^{\prime}>s$. Since $d_{i}^{\prime}<d_{i} \leq r$, the first nonzero gap in $d^{\prime}$ (the external gap) occurs before $i$. For $j$ with $i \leq j \leq k$, using the properties of internal gaps shown above, we conclude that $d_{j}^{\prime} \leq d_{i}^{\prime}-(j-i) g+1$. Meanwhile, $d_{j} \geq d_{i}-(j-i) g$; therefore, $d_{j}^{\prime} \leq d_{j}$. For $j>k$, again $d_{j}^{\prime}=s \leq d_{j}$. These inequalities imply that the sum of terms in $d$ exceeds the sum in $d^{\prime}$, which contradicts $d, d^{\prime} \in P_{m, n, r, s, g}$.

Including the external gaps, the unique maximal element of $P_{m, n, r, s, g}$ has at most three gaps that are less than $g$. We next reduce the problem of proving that the length condition suffices for feasibility to proving it for $g$-uniform lists. Recall that a list in $P_{m, n, r, s, g}$ is $g$ uniform if every nonzero gap except possibly the last equals $g$.

Definition 2.3. From a list $d \in D_{n}(r, s, g)$ whose internal gaps equal $g$ except perhaps for one $g-1$, we define $g$-uniform lists $d^{+}, d^{-} \in D_{n}(r, s, g)$. Let $d^{+}=d^{-}=d$ when $d$ has at most one nonzero gap. Otherwise, let the external gaps in $d$ be $a$ and later $b$, and let $c=a+b$.

If every internal gap in $d$ is $g$, then define $d^{+}$from $d$ by adding $a$ to each $d_{i}$ such that $r>d_{i}>s$, except that when $c>g$ also add $c-g$ to the first copy of $s$. Define $d^{-}$from $d$ by subtracting $g-a$ from each $d_{i}$ such that $r>d_{i}>s$, except that when $c<g$ subtract only $b$ from the last entry before the first copy of $s$, making it equal to $s$.

If $d$ has an internal gap of $g-1$ at some position $j$, then first form $\hat{d}^{+}$by adding 1 to each $d_{i}$ such that $i \leq j$ and $r>d_{i}$, and form $\hat{d}^{-}$by subtracting 1 from each $d_{i}$ such $i>j$ and $d_{i}>s$. Now all internal gaps in $\hat{d}^{+}$and $\hat{d}^{-}$equal $g$. Form $d^{+}$from $\hat{d}^{+}$in the way that $d^{+}$is formed from $d$ above, and form $d^{-}$from $\hat{d}^{-}$in the way that $d^{-}$is formed from $d$ above.

These lists are illustrated below, with $c=a+b$.

list case change values

\begin{tabular}{|c|c|c|c|c|c|c|c|c|}
\hline$d$ & no $g-1$ & & $\ldots, r$ & $r-a$ & $r-a-g, \ldots$ & $s+b+g$ & $s+b$ & $s, \ldots$ \\
\hline$d^{+}$ & $a+b \leq g$ & $+a$ & $\ldots, r$ & $r$ & $r-g, \ldots$ & $s+c+g$ & $s+c$ & $s, \ldots$ \\
\hline$d^{+}$ & $a+b>g$ & exception & $\ldots, r$ & $r$ & $r-g, \ldots$ & $s+c+g$ & $s+c$ & $-g$, \\
\hline$d^{-}$ & $a+b \geq g$ & $-(g-a)$ & $\ldots, r$ & $r-g$ & $r-2 g, \ldots$ & \multicolumn{2}{|c|}{$s+c, s+c-g$} & $s,$. \\
\hline$d^{-}$ & $a+b<g$ & exception & $\ldots, r$ & $r-g$ & $r-2 g, \ldots$ & $s+c$ & $s$ & $s,$. \\
\hline$d$ & $g-1$ at $j$ & & $\ldots, r$ & $r-a$ & $r-a-g, \ldots$ & $s+b+g$ & $s+b$ & $s, \ldots$ \\
\hline$\hat{d}^{+}$ & & +1 or 0 & $\ldots, r$ & $-a+1$ & $-a+1-g, \ldots$ & $s+b+g$ & $s+b$ & $s, \ldots$ \\
\hline$\hat{d}^{-}$ & & 0 or -1 & $\ldots, r$ & $r-a$ & $r-a-g, \ldots$ & $b+g-1$ & $-b-1$ & $s,$. \\
\hline
\end{tabular}


The display above shows the construction of $d^{+}$and $d^{-}$term-by-term. When every internal gap in $d$ equals $g$, explicitly $d^{+}$and $d^{-}$are $g$-uniform (the last nonzero gap is $a+b$ or $a+b-g$ ). When $d$ has one internal gap equal to $g-1$, the intermediate lists $\hat{d}^{+}$and $\hat{d}^{-}$ eliminate that difficulty. The subsequent changes are as applied previously to a list with no such gap, so again the final lists $d^{+}$and $d^{-}$are $g$-uniform.

Our requirement that $a=g$ for a $g$-uniform list is asymmetric. The proofs could be carried through with $b=g$ instead. Choosing one alternative for the definition of $g$-uniform reduces the set of lists we need to test to prove the length threshold.

Lemma 2.4. Let $d$ be the maximal element of $P_{m, n, r, s, g}$. If the $g$-uniform lists $d^{+}$and $d^{-}$ formed from d in Definition 2.3 are both feasible, then $d$ is feasible.

Proof. Suppose first that every internal gap of $d$ is $g$. For each $k$, we compare $L_{k}(d)$ and $R_{k}(d)$ using $L_{k}\left(d^{+}\right) \leq R_{k}\left(d^{+}\right)$and $L_{k}\left(d^{-}\right) \leq R_{k}\left(d^{-}\right)$. If $d_{k}=r$, then $L_{k}(d)=L_{k}\left(d^{-}\right) \leq$ $R_{k}\left(d^{-}\right) \leq R_{k}(d)$. If $d_{k}=s$, then $R_{k}(d)=R_{k}\left(d^{+}\right) \geq L_{k}\left(d^{+}\right) \geq L_{k}(d)$.

Hence we may assume $s<d_{k}<r$. Let $t=\left|\left\{i: d_{k} \leq d_{i}<r\right\}\right|$; each index $i$ counted here is at most $k$, since each internal gap is nonzero. Let $t^{\prime}=\mid\left\{i: i>k\right.$ and $\left.s<d_{i}<k\right\} \mid$. Note that $t$ and $t^{\prime}$ count disjoint sets of indices. If $i>k$ and $d_{i} \geq k$, then the index $i$ is not counted by $t$ or $t^{\prime}$.

We compare contributions to the $k$ th E-G inequality. Since $d_{k}>s$, the computation of $L_{k}\left(d^{+}\right)$is not affected by the exception when $a+b>g$. The computation of $L_{k}\left(d^{-}\right)$is affected only when $d_{k}$ is the last term before the first $s$ and $b<g-a$; in that case the difference is smaller by $g-a-b$, and we can incorporate this by writing an inequality.

$$
\begin{aligned}
L_{k}\left(d^{+}\right)=L_{k}(d)+t a ; & L_{k}\left(d^{-}\right) \geq L_{k}(d)-t(g-a) ; \\
R_{k}\left(d^{+}\right) \leq R_{k}(d)+\left(t^{\prime}+\epsilon^{+}\right) a ; & R_{k}\left(d^{-}\right) \leq R_{k}(d)-\left(t^{\prime}-\epsilon^{-}\right)(g-a) .
\end{aligned}
$$

To handle the exceptions in Definition 2.3, we set $\left(\epsilon^{+}, \epsilon^{-}\right)$to $(1,0)$ if $a+b>g$, to $(0,1)$ if $a+b<g$, and to $(0,0)$ if $a+b=g$. Before considering that, the reason for the inequality bounding $R_{k}\left(d^{+}\right)$is that $\min \left\{k, d_{i}^{+}\right\}$is taken before contributing to $R_{k}$. For $R_{k}\left(d^{-}\right)$, the contribution from each index counted by $t^{\prime}$ decreases by $g-b$, and an entry with $i>k$ and $d_{i} \geq k$ may contribute less to $R_{k}\left(d^{-}\right)$than to $R_{k}(d)$. For the exceptions, if $a+b>g$, then $R_{k}\left(d^{+}\right)$is larger by $a+b-g$, which is at most $a$. If $a+b<g$, then $R_{k}\left(d^{-}\right)$is larger by $g-a-b$, which is less than $g-a$.

These computations and the feasibility of $d^{+}$and $d^{-}$yield two upper bounds on $L_{k}(d)$ :

$$
\begin{aligned}
& L_{k}(d)=L_{k}\left(d^{+}\right)-t a \leq R_{k}\left(d^{+}\right)-t a \leq R_{k}(d)+\left(t^{\prime}-t+\epsilon^{+}\right) a ; \\
& L_{k}(d) \leq L_{k}\left(d^{-}\right)+t(g-a) \leq R_{k}\left(d^{-}\right)+t(g-a) \leq R_{k}(d)+\left(t-t^{\prime}+\epsilon^{-}\right)(g-a) .
\end{aligned}
$$


Both inequalities bound $L_{k}(d)$, and always one of the additive constants is nonpositive, since only one of $\left\{\epsilon^{+}, \epsilon^{-}\right\}$can be positive. Hence $L_{k}(d) \leq R_{k}(d)$, and we conclude that $d$ is feasible.

Now suppose that every internal gap of $d$ is $g$ except for one $g-1$ at position $j$. Recall that $\hat{d}^{+}$is formed from $d$ by adding 1 to each $d_{i}$ such that $i \leq j$ and $r>d_{i}$. Let us call the process in Definition 2.3 that produces a $g$-uniform list by augmenting some entries by $a$ (and maybe augmenting the first $s$ by $a+b-g$ ) the augmentation procedure. By definition, we obtain $d^{+}$from $\hat{d}^{+}$using the augmentation procedure. We note that the same list results from $\hat{d}^{-}$via the augmentation procedure. Similarly, the same list $d^{-}$is obtained from both $\hat{d}^{-}$and $\hat{d}^{+}$. Since we are given that $d^{+}$and $d^{-}$are feasible, the preceding argument implies that $\hat{d}^{+}$and $\hat{d}^{-}$are feasible.

We now compare $d$ with $\hat{d}^{+}$and $\hat{d}^{-}$to show that $d$ is feasible. If $k \leq j$, then $L_{k}(d)=$ $L_{k}\left(\hat{d}^{-}\right) \leq R_{k}\left(\hat{d}^{-}\right) \leq R_{k}(d)$. If $k \geq j$, then $R_{k}(d)=R_{k}\left(\hat{d}^{+}\right) \geq L_{k}\left(\hat{d}^{+}\right) \geq L_{k}(d)$. Thus $L_{k}(d) \leq R_{k}(d)$ for all $k$, as desired.

Although $d^{+}$and $d^{-}$generally have different sum from $d$, they have the same length, maximum, minimum, and bound on gaps. Hence each satisfies the desired length threshold if and only if $d$ does. We conclude that if satisfying the length threshold suffices to make a $g$-uniform list feasible, then it is also sufficient in the larger family $D_{n}(r, s, g)$.

\section{$3 \quad$ Lists without Internal Repetitions}

To simplify our study of $g$-uniform lists, we reduce the problem of checking feasibility to checking the $\ell(d)$ th E-G inequality, where $\ell(d)=\max \left\{k: d_{k} \geq k\right\}$. In fact, we prove that for every list having at most one internal gap equal to 0 , it suffices to check only the $\ell(d)$ th E-G inequality. Furthermore, this result is sharp.

Example 3.1. For $j \geq 3$, let $d=\left(2 j, 2 j-1, \ldots, j+1, j, j, j, 1^{(j[j-3] / 2)}\right)$. The initial portion is a strictly decreasing list of $j$ terms before the double repetition. Thus $d_{j}=j+1$ and $d_{j+1}=j$, so $\ell(d)=j$. We have $L_{j}(d)=j(3 j+1) / 2$ and $R_{j}(d)=j(j-1)+3 j+j(j-3) / 2$; equality holds. Nevertheless, $L_{j-1}(d)=\left(3 j^{2}-j-2\right) / 2$ and $R_{j-1}(d)=(j-1)(j-2)+4(j-1)+j(j-3) / 2=$ $\left(3 j^{2}-j-2\right) / 2-1$, so the list is not feasible.

Similarly, for $j \geq 5$, let $d=\left(2 j-1, \ldots, j+1, j, j, j-1, j-1,1^{(j[j-5] / 2+2)}\right)$. Now there are $j-1$ terms before the first repetition, so again $\ell(d)=j$. Now $L_{j}(d)=j(3 j-1) / 2$ and $R_{j}(d)=j(j-1)+3 j-2+j(j-5) / 2+2$; equality holds. However, $L_{j-2}(d)=R_{j-2}(d)+1$, so the list is not feasible. 
Recall that in general $d$ is feasible if and only if $L_{k}(d) \leq R_{k}(d)$ for $1 \leq k \leq \ell(d)[10]$.

Theorem 3.2. Let $d$ be a nonincreasing integer list of length $n$ with largest entry $r$ and smallest entry $s$, such that $n>r$ and $d_{i+1}=d_{i}$ for at most one index with $d_{i} \notin\{r, s\}$. If $L_{\ell(d)} \leq R_{\ell(d)}$, then $d$ is feasible.

Proof. We reduce $L_{k}(d) \leq R_{k}(d)$ for $k \leq \ell(d)$ to $L_{\ell(d)} \leq R_{\ell(d)}$. If $k \leq s$, then $L_{k}(d) \leq k r \leq$ $k(n-1)=k(k-1)+(n-k) k=R_{k}(d)$, since $\min \left\{k, d_{i}\right\}=k$ for all $i$. Hence it suffices to show that $L_{k+1}(d) \leq R_{k+1}(d)$ implies $L_{k}(d) \leq R_{k}(d)$ for $k$ with $s<k<\ell(d)$.

Let $j$ be the last index such that $d_{j}>k$; since $k<\ell(d)$, we have $d_{k+1}>k$, and hence $j>k$. If $j>r$, then we use $\min \left\{d_{i}, k\right\}=k$ for $i \leq j$ to compute

$$
L_{k}(d) \leq k r \leq k(j-1)=k(k-1)+k(j-k) \leq R_{k}(d) .
$$

Now consider $j \leq r$. By the choice of $j, \sum_{i=k+2}^{n} \min \left\{k+1, d_{i}\right\}=j-k-1+\sum_{i=k+2}^{n} \min \left\{k, d_{i}\right\}$. Thus

$$
R_{k}(d)=R_{k+1}(d)-2 k+\min \left\{k, d_{k+1}\right\}-(j-k-1),
$$

which simplifies to $R_{k}(d)=R_{k+1}(d)-j+1$. Therefore,

$$
L_{k}(d)=L_{k+1}(d)-d_{k+1} \leq R_{k+1}(d)-d_{k+1}=R_{k}(d)+j-d_{k+1}-1 .
$$

If $d_{k+1}=r$, then we have $L_{k}(d) \leq R_{k}(d)$ since $j \leq r$. If $d_{k+1}<r$, then since $d_{j}>k>s$, the gaps from $k+1$ through $j-1$ are nonzero, except possibly for one. Hence $d_{k+1}-d_{j} \geq$ $j-(k+1)-1$, and thus $j-1-d_{k+1} \leq k+1-d_{j} \leq 0$, which yields $L_{k}(d) \leq R_{k}(d)$.

\section{The Length Threshold}

In Section 2, we reduced feasibility of the maximal element in $P_{m, n, r, s, g}$ to showing that two "nearby" $g$-uniform lists having the same length but different sum are feasible. Proving feasibility for $g$-uniform lists in $D_{n}(r, s, g)$ implies that all lists in $D_{n}(r, s, g)$ are feasible. Since $g$-uniform lists have no internal repetitions, Theorem 3.2 implies that for feasibility of a $g$-uniform list $d$, it suffices to check only the $\ell(d)$ th E-G inequality. In this section, we obtain a sharp threshold $h(r, s, g)$ such that if $n \geq h(r, s, g)$, then the $\ell(d)$ th inequality for a $g$-uniform list $d$ in $D_{n}(r, s, g)$ does hold.

Working backward from the first copy of $s$, the number of steps to reach the last copy of $r$ in a $g$-uniform list is $\lceil(r-s) / g\rceil$. With $x+1$ being the number of copies of $r$ and $y$ being the number of copies of $s$, we thus have $n=x+y+z$, where $z=\lceil(r-s) / g\rceil$. 
To start the proof, we eliminate easy cases for the value of $\ell(d)$. Recall that the reduction to the $\ell(d)$ th inequality (Theorem 3.2) requires $n>r$, which is equivalent to the condition $L_{1}(d) \leq R_{1}(d)$.

Lemma 4.1. For $n>r$, if $d \in D_{n}(r, s, g)$, then $d$ is feasible unless $x<\ell(d) \leq n-y$.

Proof. If $x \geq r$, then $d_{r+1}=r$ and $\ell(d)=r$. Since it suffices to prove the $k$ th inequality, where $k \leq \ell(d)=r$, we have $L_{k}(d)=k r=k(k-1)+(r+1-k) k \leq k(k-1)+(n-k) k \leq R_{k}(d)$, since $\min \{k, r\}=k$. Hence $d$ is feasible unless $x<r$. In this case $d_{x+1}=r>x$, which yields $\ell(d)>x$.

As we remarked in proving Theorem 3.2, the $k$ th E-G inequality holds whenever $k \leq s$ (if $n>r$ ). Hence we have feasibility unless $\ell(d)>s$, which requires $d_{s+1}>s$. Hence the number of copies of $s$ is less than $n-s$; that is, $y<n-s$. Since $d_{n-y+1}=s<n-y+1$, we have $\ell(d) \leq n-y$.

We remark that the conditions of Lemma 4.1 cannot be weakened when $n=r+1$ and $r>s$, since the lists $\left(r^{(r)}, r-1\right)$ and $\left(r^{(s+1)}, s^{(r-s)}\right)$ are not feasible. We can now obtain the length threshold for feasibility.

Theorem 4.2. Given $r, s, g \in \mathbb{N}$ with $r>s$, let $z=\lceil(r-s) / g\rceil$ and $b=r-s-g(z-1)$. If $n \geq h(r, s, g)$, then every list in $D_{n}(r, s, g)$ is feasible, where

$$
h(r, s, g)=\frac{1}{s}\left(\left\lfloor\frac{(r+s)^{2}}{4}\right\rfloor+\left\lceil\frac{r+s}{2}\right\rceil-b\left\lfloor\frac{z}{2}\right\rfloor+\frac{g z}{2}-\frac{g}{2}\left\lceil\frac{z^{2}}{2}\right\rceil\right) .
$$

Furthermore, the bound is sharp; $D_{n}(r, s, g)$ has an infeasible list when $n=\lceil h(r, s, g)\rceil-1$.

Proof. By Lemma 2.4, it suffices to determine the threshold on $n$ so that the $g$-uniform lists in $D_{n}(r, s, g)$ are feasible. There are $z$ nonzero gaps, and only the last can fail to be $g$; it equals $b$. For $g$-uniform lists, we have reduced the checking of feasibility to checking the $\ell(d)$ th E-G inequality (by Theorem 3.2). By Lemma 4.1, we may assume that $x<\ell(d) \leq n-y$, where $d$ has $x+1$ copies of $r$ and $y$ copies of $s$.

Given the parameters $n, r, s, g$, a $g$-uniform list in $D_{n}(r, s, g)$ is completely determined by specifying $x$; hence specifying $x$ also determines $\ell(d)$. We henceforth abbreviate $\ell(d)$ to $\ell$ and think of $\ell, L_{\ell}$, and $R_{\ell}$ as functions of $x$. Our proof is in three steps: we find the value of $\ell$ such that the $\ell$ th $E-G$ inequality is hardest to satisfy (meaning that if that one holds then they all hold), determine the value of $x$ that yields that value of $\ell$, and finally determine the threshold length where that inequality holds. 
To facilitate the explanation of the argument, we illustrate the critical situation in Figure 1 ; the height of the $i$ th column is $d_{i}$. The data is $(r, s, g)=(19,3,5)$, which produces $(z, b)=(4,1)$. The critical choices are $(\ell, x)=(12,10)$, and the threshold for $n$ is 26 .

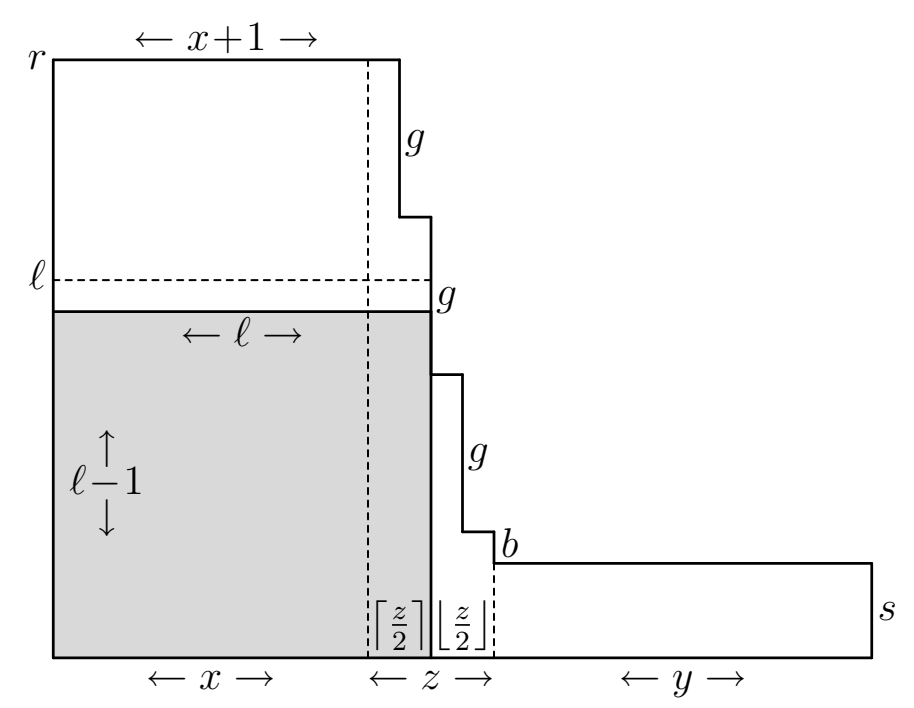

Figure 1: A g-uniform list (at the threshold)

Step 1: For each $n$, the inequality $L_{\ell} \leq R_{\ell}$ is hardest to satisfy when $\ell=\lceil(r+s) / 2\rceil$. As noted, Lemma 4.1 allows us to assume that $x<\ell \leq n-y$ (ignore for now that $\ell$ is drawn as $x+\lceil z / 2\rceil$ in Figure 1). By the definition of $\ell$, we have $d_{i} \geq \ell$ for $i \leq \ell$ and $d_{i} \leq \ell$ for $i>\ell$. Thus $L_{\ell}$ is the area of the diagram in and above the gray box, while $R_{\ell}$ is the area of the diagram in and to the right of the gray box. The list is determined by choosing $x$; changing $x$ moves the staircase and also moves $\ell$. We study the change in $R_{\ell}-L_{\ell}$ as $x$ changes.

Both $L_{\ell}$ and $R_{\ell}$ count the area of the gray box; the difference cancels it. The remainder of $L_{\ell}$ is an $(r-\ell+1)$-by- $\ell$ rectangle with an arithmetic sum missing. The remainder of $R_{\ell}$ is an $y$-by-s rectangle plus an arithmetic sum. That is,

$$
R_{\ell}-L_{\ell}=y s+(x+z-\ell)(s+b)+g\left(\begin{array}{c}
x+z-\ell \\
2
\end{array}\right)-(r-\ell+1) \ell+g\left(\begin{array}{c}
\ell-x \\
2
\end{array}\right) .
$$

To prove that $R_{\ell}-L_{\ell}$ is minimized when $x$ is chosen to make $\ell=\lceil(r+s) / 2\rceil$, we begin with a formula for $\ell$ in terms of $x$. Under the condition $x<\ell \leq n-y$, the value of $\ell$ is the largest $i$ such that $r-g(i-x-1) \geq i$. This simplifies to $i \leq \frac{r+g(x+1)}{g+1}$, and thus $\ell=\left\lfloor\frac{r+g(x+1)}{g+1}\right\rfloor$. Note that when $x$ increases by 1 , usually $\ell$ increases by 1 , but when $r+g(x+1) \equiv 0 \bmod (g+1)$, the value of $\ell$ is the same for $x$ and $x+1$.

When increasing $x$ by 1 also increases $\ell$, the only terms in the formula in (1) that change are $-(r-\ell+1) \ell$ and $y s$ (since $n$ and $z$ are fixed, $x+y$ is fixed). Hence the change is $2 \ell-r-s$. 
When $\ell(x+1)=\ell(x)$, the other terms change. Since $x+z-\ell$ increases and $\ell-x$ decreases, the change is $-s+(s+b)+g(x+z-\ell)-g(\ell-x-1)$, which simplifies to $b+g(z+2 x-2 \ell+1)$. Since $z g=r-s-b+g$, we can rewrite this as $r-s+2 g(x-\ell+1)$. The condition $\ell(x+1)=\ell(x)$ occurs when $r+g(x+1) \equiv 0 \bmod (g+1)$, so $\ell=\frac{r+g(x+1)}{g+1}$, and $x+1-\ell=(\ell-r) / g$. Thus again the change is $2 \ell-r-s$.

When $x$ is small, $R_{\ell}-L_{\ell}$ decreases as $x$ increases until $\ell$ reaches $\lceil(r+s) / 2\rceil$. Thereafter, $R_{\ell}-L_{\ell}$ increases as $x$ continues to increase. Hence for fixed $n$ all $g$-uniform lists are feasible if and only if the list obtained by choosing $x$ to produce $\ell=\lceil(r+s) / 2\rceil$ is feasible.

Step 2: Setting $x=\lceil(r+s) / 2\rceil-\lceil z / 2\rceil$ yields $\ell(x)=\lceil(r+s) / 2\rceil$. Since $\ell$ increases by 0 or 1 as $x$ increases, some choice of $x$ produces $\ell(x)=\lceil(r+s) / 2\rceil$. Let $\lambda=\lceil(r+s) / 2\rceil$, and set $x=\lambda-\lceil z / 2\rceil$. We show that $d_{\lambda} \geq \lambda$ and $d_{\lambda+1}<\lambda+1$ for this choice of $x$. Recall that $d_{i}=r-g(i-x-1)$ for $x<i \leq x+z$. Since $\lambda=x+\lceil z / 2\rceil$ and $g z=r-s+g-b$,

$$
\begin{aligned}
d_{\lambda} & =r-g(\lceil z / 2\rceil-1) \geq r-g(z-1) / 2 \\
& =r-(r-s+g-b) / 2+g / 2=(r+s) / 2+b / 2 \geq \lambda .
\end{aligned}
$$

Similarly, if $\lambda+1 \leq x+z$, then

$$
\begin{aligned}
d_{\lambda+1} & =r-g\lceil z / 2\rceil \leq r-g z / 2 \\
& =r-(r-s+g-b) / 2=(r+s) / 2-(g-b) / 2<\lambda+1 .
\end{aligned}
$$

The inequality $\lambda+1 \leq x+z$ fails if and only if $z=1$. In this case, $r-s=g$ and $\lambda=x+1$. Since $r>s$, we have $x=\lambda-1=\lceil(r+s) / 2\rceil-1 \geq s$. Hence $d_{\lambda+1}=s \leq x<x+2=\lambda+1$. In both cases, we obtain $d_{\lambda+1}<\lambda+1$. We have therefore shown that $\ell=\lambda=\lceil(r+s) / 2\rceil$.

Step 3: $n \geq h(r, s, g)$ is sufficient for feasibility. Having reduced the problem to studying the unique $g$-uniform list of length $n$ with $x=\lceil(r+s) / 2\rceil-\lceil z / 2\rceil$ and $\ell=\lceil(r+s) / 2\rceil$ (see Figure 1), it suffices to determine the threshold on $n$ such that $R_{\ell}-L_{\ell} \geq 0$. We simplify (1) using $y=n-x-z=n-\ell+\lfloor z / 2\rfloor, r-\ell=\lfloor(r-s) / 2\rfloor$, and $\ell-x=\lceil z / 2\rceil$ to obtain

$$
\begin{aligned}
& R_{\ell}-L_{\ell}=\left(n-\ell-\left\lfloor\frac{z}{2}\right\rfloor\right) s+\left\lfloor\frac{z}{2}\right\rfloor(s+b)+g\left(\begin{array}{c}
\lfloor z / 2\rfloor \\
2
\end{array}\right)-\left(\left\lfloor\frac{r-s}{2}\right\rfloor+1\right)\left\lceil\frac{r+s}{2}\right\rceil+g\left(\begin{array}{c}
\lceil z / 2\rceil \\
2
\end{array}\right) \\
& =n s-\left\lceil\frac{r+s}{2}\right\rceil\left(\left\lfloor\frac{r+s}{2}\right\rfloor+1\right)+b\left\lfloor\frac{z}{2}\right\rfloor-\frac{g z}{2}+\frac{g}{2}\left(\left\lfloor\frac{z}{2}\right\rfloor\left\lfloor\frac{z}{2}\right\rfloor+\left\lceil\frac{z}{2}\right\rceil\left\lceil\frac{z}{2}\right\rceil\right) \\
& =n s-\left\lfloor\frac{(r+s)^{2}}{4}\right\rfloor-\left\lceil\frac{r+s}{2}\right\rceil+b\left\lfloor\frac{z}{2}\right\rfloor-\frac{g z}{2}+\frac{g}{2}\left\lceil\frac{z^{2}}{2}\right\rceil \quad=n s-\operatorname{sh}(r, s, g) \text {. }
\end{aligned}
$$

We conclude that if $n \geq h(r, s, g)$, then every list in $D_{n}(r, s, g)$ is feasible. 
Step 4: The bound is sharp; that is, when $n=\lceil h(r, s, g)\rceil$ there is an infeasible list in $D_{n-1}(r, s, g)$. Since $z=\lceil(r-s) / g\rceil$, always $z \leq r-s$. With $x$ set to $\lceil(r+s) / 2\rceil-\lceil z / 2\rceil$, we thus have $x+z=\lceil(r+s) / 2\rceil+\lfloor z / 2\rfloor \leq r$.

Since every list in $D_{n}(r, s, g)$ is feasible when $n=\lceil h(r, s, g)\rceil$, the first E-G inequality requires $\lceil h(r, s, g)\rceil \geq r+1$. Furthermore, all lists with length at most $r$ are infeasible. Therefore, we may assume that $\lceil h(r, s, g)\rceil \geq r+2$. Since $x+z \leq r$, the key $g$-uniform list at the threshold length has at least two copies of $s$. Hence the list obtained by deleting one copy of $s$ belongs to $D_{n-1}(r, s, g)$ and is infeasible.

The expression for the threshold length simplifies when $g \mid(r-s)$, in which case $b=g$.

Corollary 4.3. For $g \mid(r-s)$, the threshold length for feasibility of all lists in $D_{n}(r, s, g)$ is

$$
\frac{1}{s}\left(\left\lfloor\frac{(r+s)^{2}}{4}\right\rfloor+\left\lceil\frac{r+s}{2}\right\rceil-\frac{g}{2}\left\lfloor\frac{(r-s)^{2}}{2 g^{2}}\right\rfloor\right) .
$$

When $g=1$, this simplifies to $r+\frac{r+s+\epsilon}{2 s}$, with $\epsilon=0$ for even $r+s$ and $\epsilon=1$ for odd $r+s$. If $g=r-s$ (and hence there is no gap constraint), then it simplifies to $\frac{(r+s+1)^{2}-\epsilon^{\prime}}{4 s}$, where $\epsilon^{\prime}=0$ if $r+s$ is odd and $\epsilon^{\prime}=1$ if $r+s$ is even. Furthermore, the thresholds are sharp.

Proof. For the first statement, set $b=g$ and $z=(r-s) / g$. For the second and third, set $g=1$ or $z=1$. Sharpness was proved in greater generality in Theorem 4.2.

Finally, we return to our motivating question about the threshold length for even-summed lists to be graphic. It may happen that our infeasible list with $n=\lceil h(r, s, g)\rceil-1$ has odd sum. If all infeasible lists with that length have odd sum, then the threshold will be $\lceil h(r, s, g)\rceil-1$. Before exhibiting a family where this occurs, we show that the threshold never declines by more than 1 .

Theorem 4.4. For all $r, s, g$, the least $n$ such that all even-summed lists in $D_{n}(r, s, g)$ are graphic is $\lceil h(r, s, g)\rceil$ or $\lceil h(r, s, g)\rceil-1$.

Proof. Let $m=\lceil h(r, s, g)\rceil$. Since every list of length $r$ fails the first E-G inequality, having length $m-2$ be sufficient for even-summed lists to be graphic requires $\lceil h(r, s, g)\rceil \geq r+3$. Since we have noted that $x+z \leq r$ at the key value of $x$, the key $g$-uniform list with length $m$ has at least three copies of $s$.

Deleting one copy of $s$ yields an infeasible list $d$ in $D_{m-1}(r, s, g)$, meaning that $L_{\ell}(d)-$ $R_{\ell}(d) \geq 1$. Deleting another copy of $s$ still retains a copy of $s$ and hence yields an infeasible list $d^{\prime}$ in $D_{m-2}(r, s, g)$ with $L_{\ell}\left(d^{\prime}\right)-R_{\ell}\left(d^{\prime}\right) \geq s+1 \geq 2$. 
Since $z=\lceil(r-s) / g\rceil \leq r-s$, we have $x=\lceil(r+s) / 2\rceil-\lceil z / 2\rceil \geq\lceil(r+s) / 2\rceil-$ $\lceil(r-s) / 2\rceil=s$. Hence there are more than $s$ copies of $r$ in $d^{\prime}$. If $d^{\prime}$ has even sum, then $d^{\prime}$ is the desired infeasible list. If $d^{\prime}$ has odd sum, then we decrease the last copy of $r$ by 1 to obtain a infeasible list with length $\lceil h(r, s, g)\rceil-2$ that has even sum.

Determining when the threshold length for feasibility of even-summed lists in $D_{n}(r, s, g)$ equals $\lceil h(r, s, g)\rceil$ is messy, because attention must be paid to the exact value of $L_{\ell}-R_{\ell}$ at the key value of $x$. In lieu of discussing that, we close with an example of a family where the length threshold for even-summed lists to be graphic is less than the threshold for feasibility. We will use the Havel-Hakimi Theorem [6,7], which states that a list $d$ with even sum is graphic if and only if the list $d^{\prime}$ obtained from it by deleting a largest entry $k$ and subtracting 1 from $k$ largest remaining entries is graphic. Call that operation a Havel-Hakimi step.

Theorem 4.5. For $n \geq\lceil h(r, 1,1)\rceil-1$, every even-summed list in $D_{n}(r, 1,1)$ is graphic.

Proof. From Corollary 4.3, $h(r, 1,1)=\lfloor 3 r / 2\rfloor+1$. Nevertheless, we prove that already length $\lfloor 3 r / 2\rfloor$ is sufficient. (Theorem 4.4 implies that no smaller length is sufficient.)

Consider $d \in D_{n}(r, 1,1)$ with even sum. We use induction on $r$. For $r \leq 2$, lists with even sum and length at least $\lfloor 3 r / 2\rfloor$ are graphic. Now consider $r>2$. Let $d^{\prime}$ be the list obtained by applying a Havel-Hakimi step to $d$. Note that $d_{1}^{\prime} \in\{r, r-1, r-2\}$ and that $d^{\prime}$ is gap-free. If $d_{1}^{\prime}=r$, then $d_{r+1}=r$ and the first computation in Lemma 4.1 shows that $d$ is graphic. Note that $d$ has at least $r-2$ distinct values between $r$ and 1 . Hence if $d_{1}^{\prime}=r-2$, then $d_{2}=r-1$ and $d^{\prime} \in D_{n^{\prime}}(r-2,1,1)$ with $n^{\prime} \in\{n-1, n-2, n-3\}$. Since $n^{\prime} \geq n-3 \geq\left\lfloor\frac{3 r}{2}\right\rfloor-3=\left\lfloor\frac{3(r-2)}{2}\right\rfloor$, the induction hypothesis implies that $d^{\prime}$ is graphic.

Now suppose $d_{1}^{\prime}=r-1$, so $d_{2}=r$ or $d_{r+2}=r-1$. If $d_{r+2}=r-1$, then $d^{\prime} \in$ $D_{n-1}(r-1,1,1)$. By the induction hypothesis, $d^{\prime}$ is graphic.

Finally, suppose $d_{1}^{\prime}=r-1$ and $d_{2}=r$. Since $d$ is gap-free, $d_{r} \geq 2$. Hence $d^{\prime} \in$ $D_{n^{\prime}}(r-1,1,1)$ with $n^{\prime} \in\{n-1, n-2\}$. If $n^{\prime}=n-1$, then $n^{\prime}=n-1 \geq\left\lfloor\frac{3 r}{2}\right\rfloor-1 \geq\left\lfloor\frac{3(r-1)}{2}\right\rfloor$, and the induction hypothesis applies. If $n^{\prime}=n-2$, then $d_{r}=2$ and $d_{r+1}=1$, and hence $d=\left(r, r, r-1, \ldots, 2,1,1^{(\lfloor r / 2\rfloor-1)}\right)$. If $r$ is odd, then the degree sum of $d$ is $2 r+\left(\begin{array}{l}r \\ 2\end{array}\right)+(r-1) / 2-1$, which is odd, so this case does not occur. If $r$ is even, then

$$
n^{\prime}=n-2 \geq\left\lfloor\frac{3 r}{2}\right\rfloor-2=\left\lfloor\frac{3(r-1)-1}{2}\right\rfloor=\left\lfloor\frac{3(r-1)}{2}\right\rfloor,
$$

and again the induction hypothesis applies.

\section{Acknowledgments}

The authors thank Erin W. Chambers and Mohit Kumbhat for helpful comments. 


\section{References}

[1] M. Aigner and E. Triesch, Realizability and uniqueness in graphs, Discrete Math. 136 (1994), 103-113.

[2] R. B. Eggleton, Graphic sequences and graphic polynomials: a report. Infinite and finite sets (Colloq., Keszthely, 1973; dedicated to P. Erdös on his 60th birthday), Vol. 1, pp. 385-392. Colloq. Math. Soc. Janos Bolyai, 10 (North-Holland, Amsterdam, 1975).

[3] P. Erdős and T. Gallai, Graphs with prescribed degree of vertices (Hungarian), Mat. Lapok 11 (1960), 264-274.

[4] S. Y. R. Li, Graphic sequences with unique realization, J. Combinatorial Theory Ser. B 19 (1975), 42-68.

[5] P. L. Hammer, T. Ibaraki, and B. Simeone, Threshold sequences, SIAM J. Alg. Disc. Methods 2 (1981), 39-49.

[6] V. Havel, A remark on the existence of finite graphs, Casopis Pest. Mat. 80 (1955), $477-480$.

[7] S. L. Hakimi, On the realizability of a set of integers as degrees of the vertices of a graph, SIAM J. Appl. Math. 10 (1962), 496-506.

[8] A. Tripathi, and S. Vijay, A note on a theorem of Erdős and Gallai. Discrete Math. 265 (2003), 417-420.

[9] D. B. West, Exercise 1.3.57, Introduction to Graph Theory, 2nd ed., (Prentice-Hall, 2001), 52 .

[10] I. E. Zverovich and V. E. Zverovich, Contributions to the theory of graphic sequences, Discrete Math. 105 (1992), 293-303. 